\title{
Extranodal natural killer/T-cell lymphoma in Malawi: a report of three cases
}

\author{
Tamiwe Tomoka ${ }^{1,3^{*}}$, Eric Powers ${ }^{2}$, Toon van der Gronde ${ }^{1}$, Amy Amuquandoh', Bal Mukunda Dhungel', \\ Coxcilly Kampani ${ }^{1}$, Steve Kamiza ${ }^{3}$, Nathan D. Montgomery², Yuri Fedoriw ${ }^{2}$ and Satish Gopal ${ }^{1,2,3}$
}

\begin{abstract}
Background: Extranodal NK/T-cell lymphoma (ENKTCL) reports from sub-Saharan Africa (SSA) are remarkably rare, despite early childhood acquisition and high prevalence of the causative infectious agent, Epstein-Barr virus (EBV), and frequent occurrence of other lymphoproliferative disorders causally associated with EBV.

Case presentations: At a national teaching hospital in Malawi, three patients of African descent were seen with ENKTCL between 2013 and 2014. Patients were aged between 29 and 60 years, two with craniofacial involvement and one with a primary abdominal tumor, and all were HIV-negative. All had systemic B symptoms, and two severely impaired performance status. On histologic review, morphology and immunophenotyping demonstrated classical ENKTCL features in all cases, including diffuse proliferations of intermediate-to-large atypical lymphocytes with high mitotic activity and extensive background necrosis, positivity for CD3 and CD56, and negativity for CD20. By in situ hybridization, all three tumors were positive for EBV-encoded RNA (EBER). Baseline plasma EBV DNA was also markedly elevated for all three patients. Due to radiotherapy and chemotherapy limitations, patients were treated with CHOP (cyclophosphamide, doxorubicin, vincristine, prednisone) with rapid disease progression. All three patients died from progressive lymphoma within 3 months of initial diagnosis.
\end{abstract}

Conclusions: Our experience with these three patients in Malawi can highlight that ENKTCL does indeed occur in SSA, increase familiarity with ENKTCL among clinicians and pathologists throughout the region, and emphasize the need for better diagnosis and treatment for this neglected population.

Keywords: Non-Hodgkin lymphoma, Epstein-Barr virus, Sub-Saharan Africa

\section{Background}

It is vital to improve lymphoma diagnostic infrastructure in sub-Saharan Africa (SSA) to fully understand the heterogeneity of non-Hodgkin lymphoma (NHL) in the region, provide better prognostic information to patients and clinicians, and allow more subtype-directed therapy where appropriate. In resource-rich settings, molecular profiling is increasingly leading to tailored therapeutic approaches even within well-defined histologic categories, like diffuse large B-cell lymphoma. However, due to resource limitations in SSA, the entire spectrum of NHL is often diagnosed and treated as a single entity. Among low- and middle-income countries, extranodal NK/T-cell lymphoma (ENKTCL) in particular has been principally

\footnotetext{
* Correspondence: ttomoka@unclilongwe.org

${ }^{1}$ UNC Project-Malawi, Private Bag A104, Lilongwe, Malawi

${ }^{3}$ University of Malawi College of Medicine, Blantyre, Malawi

Full list of author information is available at the end of the article
}

described in Asia and Latin America, and published reports from SSA are remarkably few. This is despite early childhood acquisition and high prevalence of the causative infectious agent, Epstein-Barr virus (EBV), in SSA, as well as frequent occurrence of other lymphoproliferative disorders that are causally associated with EBV, like endemic Burkitt lymphoma and classical Hodgkin lymphoma. Therefore, to increase regional awareness of this disease, highlighting the occurrence of ENKTCL specifically in SSA is important.

\section{Case presentations}

At a national teaching hospital in the capital of Malawi, Lilongwe, three patients of African descent were seen with ENKTCL between September 2013 and March 2014, and were enrolled in the prospective Kamuzu Central Hospital Lymphoma Study cohort after informed consent. Case 1 was a 29-year-old male with a greater than 6 month 
history of an abdominal mass. Case 2 was a 51 -yearold male who presented with a 4 month history of a sinonasal tumor eroding the medial wall of the maxilla associated with right eye proptosis. Case 3 was a 60-year-old female with a less than 3 month history of progressive left maxillary sinus mass involving the medial left and right orbits. All three patients were HIV-negative, all had systemic B symptoms, and two had severely impaired Eastern Cooperative Oncology Group performance status of greater than 2 .

Biopsy and tumor characterization using immunohistochemistry performed locally in Lilongwe was done for all patients, and was reviewed in real time during a weekly telepathology conference conducted between clinicians and pathologists in Lilongwe and Chapel Hill, as previously described in detail [1]. Histologically, all three cases showed diffuse proliferations of intermediate-to-large atypical lymphocytes with high mitotic activity and areas of extensive background necrosis, as shown for a single case exhibiting features typical of all three in the accompanying Fig. 1. Immunophenotypically, the tumors were all positive for CD3 and CD56, and negative for CD20. Subsequent in situ hybridization (ISH) in Chapel Hill demonstrated all three tumors to be positive for EBV-encoded RNA (EBER). All patients had staging bone marrow evaluations without involvement by lymphoma.
Other baseline laboratory investigations were normal excepting mild anemia (hemoglobin $10.8 \mathrm{~g} / \mathrm{dL}$ ) in one case, and elevated lactate dehydrogenase in two cases. Baseline plasma EBV DNA was also markedly elevated for all three patients by real-time quantitative polymerase chain reaction, ranging from 3412 to $2,098,940$ copies/mL. Radiotherapy is currently not available anywhere in Malawi, and limited supportive care infrastructure and low drug availability prohibit intensive chemotherapy regimens including asparaginase or high-dose methotrexate. Therefore, the local standard of care for most NHL subtypes is CHOP (cyclophosphamide, doxorubicin, vincristine, prednisone). Of our three patients, one was too sick to initiate cytototoxic treatment and two received a single dose of CHOP with rapid subsequent disease progression. All three patients died from progressive lymphoma within 3 months of initial diagnosis.

\section{Discussion and conclusions}

ENKTCL is an uncommon subtype of the the mature T and NK-cell neoplasms, a heterogeneous group of aggressive NHL that account for approximately $12 \%$ of all NHL in the United States, Europe, and Asia [2]. ENKTCL typically involves the upper aerodigestive tract, with the nasal cavity being the prototypic site of

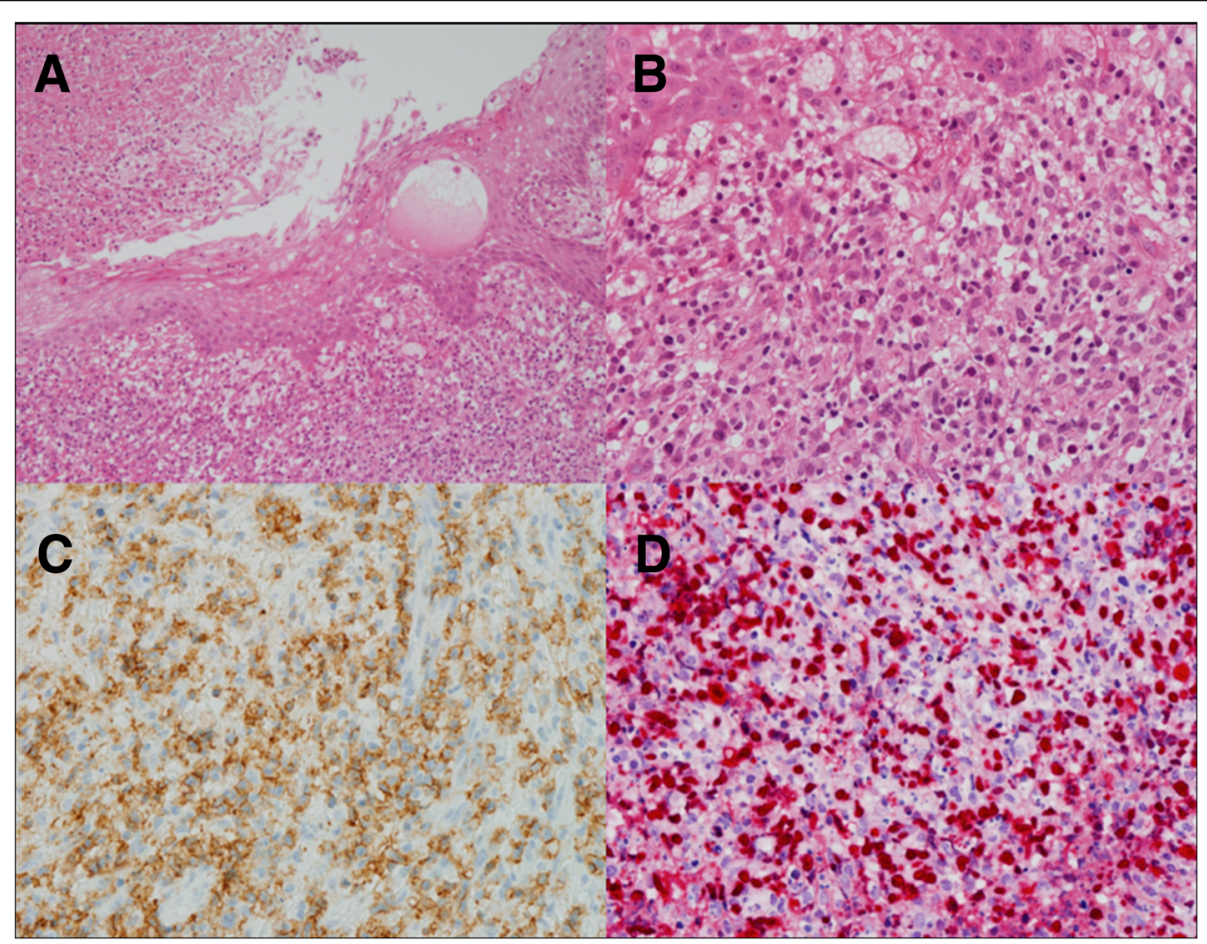

Fig. 1 Biopsy of sinonasal mass from Case 2. a Ulcerated squamous mucosa surrounded by necrosis and subepithelial proliferation of tumor cells (haematoxylin and eosin, 10x). b Moderate-to-large atypical lymphoid-appearing tumor cells with high mitotic activity (haematoxylin and eosin, 40x). c Tumor cells positive for CD56 by immunohistochemistry (40x). d Tumor cells positive for Epstein-Barr virus-encoded RNA by in situ hybridization (40x) 
involvement. Sites for extranasal involvement include skin, soft tissue, gastrointestinal tract, and testes [3]. Our cases highlight the rapid clinical progression of this disease and short overall survival, particularly after suboptimal treatment as in our setting. Importantly, $\mathrm{CHOP}$ was administered in our setting due to lack of more suitable alternatives like aparaginase or high-dose methotrexate, and poor outcomes after CHOP for ENKTCL are have been attributed to tumor expression of multidrug resistance protein [4].

There is significant ethnic and regional variation in ENKTL prevalence worldwide. It is most common in East and Southeast Asia, as well as regions of Central and South America, where it has been reported to represent $6-15 \%$ of NHL cases [5-7]. Frequency in SSA and persons of African descent is not extensively described, but is presumed to be low, although this may reflect under-recognition and under-diagnosis given well documented diagnostic limitations in the region. Retrospective epidemiologic studies in the United States have found non-Hispanic black persons to account for 2 to $5 \%$ of all ENKTCL cases [8]. There are also a very small number of published reports of ENKTCL in SSA [9-11], in addition to other studies describing NHL of the nasal cavity and nasopharynx without complete histopathologic or immunophenotypic characterization $[12,13]$.

Pathologically, ENKTCL most commonly expresses an NK cell phenotype, being positive for CD56, cytoplasmic $\mathrm{CD} 3 \varepsilon+$, without surface T-cell receptor (TCR) and TCR gene rearrangements $[2,14]$. A subset of cases may demonstrate a T-cell phenotype with clonal TCR gene rearrangements. ENKTCL is strongly associated with EBV, which can be uniformly demonstrated in tumors by EBER ISH and plasma by qPCR $[15,16]$. Plasma EBV DNA is correlated with other markers of disease severity like clinical stage and serum lactate dehydrogenase. Plasma EBV DNA levels at baseline are also associated with clinical outcomes, and serial measurement may be valuable for assessing response to treatment [16].

In conclusion, although EBV is highly prevalent in SSA with acquisition typically during early childhood, and other EBV-associated lymphoproliferative disorders including endemic Burkitt lymphoma and classical Hodgkin lymphoma are often described, ENKTCL reports from SSA are scarce, likely reflecting under-diagnosis. We hope our experience with these three patients in Malawi can serve to highlight that ENKTCL does indeed occur in SSA, increase familiarity with ENKTCL among clinicians and pathologists throughout the region, and emphasize the need for better diagnosis and treatment for this neglected population.

\section{Abbreviations}

CHOP: Cyclophosphamide, doxorubicin, vincristine, prednisone; EBER: Epstein-Barr virus-encoded RNA; EBV: Epstein-Barr virus;
ENKTCL: Extranodal NKTT-cell lymphoma; NHL: Non-Hodgkin lymphoma; SSA: Sub-Saharan Africa

\section{Acknowledgements}

We are grateful to the patients for agreeing to participate in the study and entrusting their care to our team. We also thank Wiza Kumwenda for developing the study database, the University of North Carolina Translational Pathology Laboratory for performing additional stains including extensive technical contributions from Michelle Mathews, and the UNC Vironomics Core including Dirk Dittmer and Marcia Sanders for performing plasma EBV DNA assessments. We are also grateful to leadership of Kamuzu Central Hospital, Malawi Ministry of Health, UNC Project-Malawi, and Lineberger Comprehensive Cancer Center for their support.

\section{Funding}

This work was supported by grants from the National Institutes of Health (K01TW009488, R21CA180815, and U54CA190152 to S.G.), the Medical Education Partnership Initiative (U2GPS001965), and the Lineberger Comprehensive Cancer Center (P30CA016086). The funding body had no role in the design of the study, nor collection, analysis, and interpretation of data, nor writing the manuscript.

\section{Availability of data and materials}

All data supporting these findings are presented within the manuscript. Additional data as required may be requested from the corresponding author.

\section{Authors' contributions}

$\Pi$, EP, TvdG, and AA compiled and analyzed the data. $T$ and EP wrote the first draft. CK provided technical assistance for slide preparation and immunohistochemical staining. T, BMD, SK, NDM, and YF were responsible for all pathologic interpretations. SG provided financial, clinical, and administrative support as principal investigator of the parent Kamuzu Central Hospital Lymphoma Study. All authors read and approved the final manuscript.

\section{Ethics approval and consent to participate}

All patients provided written informed consent for participation in the Kamuzu Central Hospital Lymphoma Study, which was approved by the Malawi National Health Sciences Research Committee (Federal Wide Assurance \#5976) and University of North Carolina Biomedical Institutional Review Board (Federal Wide Assurance \#4801).

\section{Consent for publication}

Written consent to publish was obtained from all patients.

\section{Competing interests}

The authors declare that they have no competing interests.

\section{Publisher's Note}

Springer Nature remains neutral with regard to jurisdictional claims in published maps and institutional affiliations.

\section{Author details}

${ }^{1}$ UNC Project-Malawi, Private Bag A104, Lilongwe, Malawi. ${ }^{2}$ University of North Carolina, Chapel Hill, USA. ${ }^{3}$ University of Malawi College of Medicine, Blantyre, Malawi.

Received: 9 March 2017 Accepted: 28 August 2017

Published online: 06 September 2017

\section{References}

1. Gopal S, Krysiak R, Liomba NG, et al. Early experience after developing a pathology Laboratory in Malawi, with emphasis on cancer diagnoses. PLoS One. 2013;8(8):e70361.

2. Jaffe ES, Harris NL, Stein $\mathrm{H}$, et al. Introduction and overview of the classification of the lymphoid neoplasms. In: Swerdlow SH, Campo E, Harris $\mathrm{NL}$, et al., editors. WHO classification of tumours of haematopoietic and lymphoid tissues. Lyon: IARC; 2008. p. 157-66.

3. Chan JKC, Quintanilla-Martinez L, Ferry JA, et al. Extranodal NKJT Iymphoma nasal type. In: Swerdlow SH, Campo E, Harris NL, et al., editors. WHO 
classification of tumours of haematopoietic and lymphoid tissues. Lyon: IARC; 2008. p. 285-8.

4. Huang WT, Huang CC, Weng SW, Eng HL. Expression of the multidrug resistance protein MRP and the lung-resistance protein LRP in nasal NKT cell lymphoma: further exploring the role of P53 and WT1 gene. Pathology. 2009:41(2):127-32.

5. Au WY, Ma SY, Chim CS, et al. Clinicopathologic features and treatment outcome of mature T-cell and natural killer-cell lymphomas diagnosed according to the World Health Organization classification scheme: a single center experience of 10 years. Ann Oncol. 2005;16(2):206-14.

6. Laurini JA, Perry AM, Boilesen E, et al. Classification of non-Hodgkin lymphoma in central and South America: a review of 1028 cases. Blood. 2012;120(24):4795-801.

7. Peh SC. Host ethnicity influences non-Hodgkin's lymphoma subtype frequency and Epstein-Barr virus association rate: the experience of a multi-ethnic patient population in Malaysia. Histopathology. 2001;38(5):458-65.

8. Adams SV, Newcomb PA, Shustov AR. Racial patterns of peripheral T-cell lymphoma incidence and survival in the United States. J Clin Oncol. 2016; 34(9):963-71.

9. Trimeche $\mathrm{M}$, et al. Prevalence of Epstein-Barr virus in non-Hodgkin's lymphomas in central region of Tunisia. Ann Pathol. 2005;25(2):95-102.

10. Kouassi YM, Vroh BT, Amelin IH, et al. A case of nasal NK/T cell lymphoma in Africa. Rev Laryngol Otol Rhinol (Bord). 2011;132(4-5):255-7.

11. Tiholoe MM, Kotu M, Khammisa RA, et al. Extranodal natural killer/T-cell lymphoma, nasal type: 'midline lethal granuloma'. a case report. Head Face Med. 2013;9:4

12. Sabageh D, Solaja TO, Olasode BJ. Malignant tumors of the upper aerodigestive tract as seen in a Nigerian tertiary health institution. Niger J Clin Pract. 2015;18(2): 231-5.

13. Onakoya PA, Adeyi OA, Nwaorgu OG, et al. Primary extranodal non-Hodgkin's lymphoma of the upper aerodigestive tract-a descriptive analysis of the pattern seen in the University College Hospital, Ibadan. Afr J Med Med Sci. 2003;32(1):59-63.

14. Hirakawa S, Kuyama M, Takahashi S, et al. Nasal and nasal-type natural killer/ T-cell lymphoma. J Am Acad Dermatol. 1999;40(2):268-72.

15. Luzi $P$, et al. Epstein-Barr virus infection in sinonasal non-Hodgkin's lymphomas. Virchows Arch. 1994;25(2):121-5.

16. Au W, Pang A, Choy C, Chim C, Kwong Y, Dc W. Quantification of circulating Epstein-Barr virus ( EBV ) DNA in the diagnosis and monitoring of natural killer cell and EBV-positive lymphomas in immunocompetent patients. Blood. 2011;104(1):243-9.

\section{Submit your next manuscript to BioMed Central and we will help you at every step:}

- We accept pre-submission inquiries

- Our selector tool helps you to find the most relevant journal

- We provide round the clock customer support

- Convenient online submission

- Thorough peer review

- Inclusion in PubMed and all major indexing services

- Maximum visibility for your research

Submit your manuscript at www.biomedcentral.com/submit

) Biomed Central 